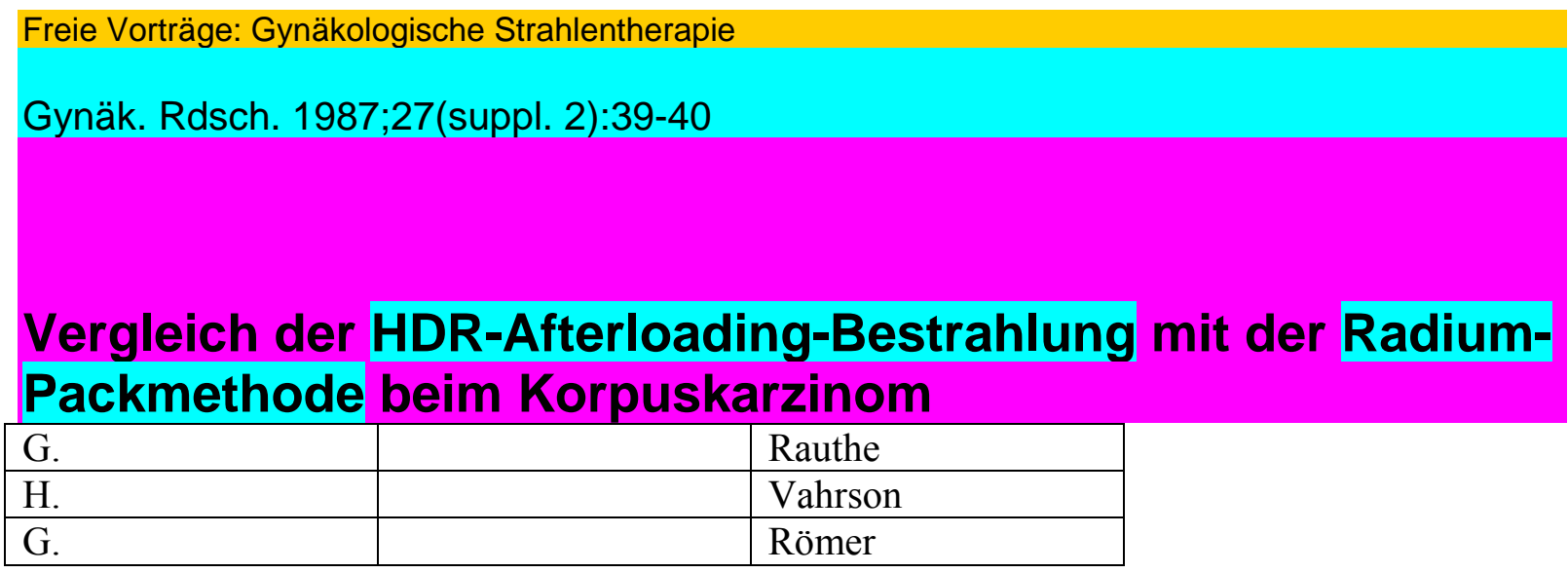

Abt. Gynäkologische Onkologie und Strahlentherapie, Zentrum für Frauenheilk. und Geburtshilfe Justus-Liebig-Universität, Gießen

Dr. G. Rauthe, Universitäts-Frauenklinik, Abteilung Gynäkologische Onkologie und Strahlentherapie, Klinikstraße 32, D6300 Gießen

Das Endometriumkarzinom ist der häufigste bösartige Tumor des weiblichen Genital-traktes in der BRD geworden, was sich auch in der Zusammensetzung des Krankengutes der UFK Gießen widerspiegelt. Seit 10 Jahren bestrahlen wir Korpuskarzinome mit einer HDR-IridiumAfterloading-Einheit Buchler. Wir stellen unsere dabei erhaltenen Ergebnisse und Komplikationen mit diesem Behandlungsverfahren vor und vergleichen sie mit denen der konventionellen LDR-Radium-Therapie in den 10 Jahren vorher.

Sowohl für die primäre als auch die postoperative Strahlentherapie entsprachen sich die

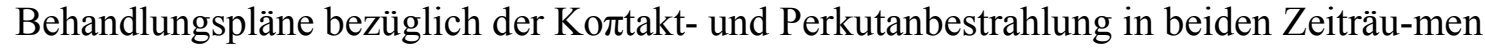
weitgehend. Während des Zeitintervalls 1977 bis 1981 änderten sich unsere HDR-Bestrahlungen mehrmals, wobei niedrigeren Einzeldosen bei höherer Fraktionierung der Vorzug gegeben wurde. Zuletzt erhielten die Patienten postoperativ bei oberflächlicher Tumorinfiltration 1-2 intravaginale Einlagen zu je 6 Gy in der A-Linie (entspr. $10 \mathrm{~Gy} /$ Applikatoroberfläche). Bei oberflächlicher Infiltration wurde die Kontaktbestrahlung durch eine biaxiale Co-60Pendelbestrahlung ergänzt, bis zu 45 Gy/B-Linie wurden appliziert (entspr. 42 Gy/A-Linie und 38 Gy/Beckenmitte). Bei defer Infiltration wurde die zusätzli-che Perkutanbestrahlung als Hochvolttherapie verabreicht bis zu 50 Gy (max. bis 60 Gy, dabei Verzicht auf Kontaktbestrahlung) homogen auf das kleine Becken.

Bei derprimären Bestrahlung im Stadium /wurde mit der HDR Afterloading-Therapie 7x6 Gy/ALinie intrauterin und 1-2 x 6 Gy/A-Linie intrazervikal verabreicht. Im Stadium II wurde auf eine Fraktion verzichtet, dafür die Kontakttherapie mit einer biaxialen Co-60-Pendelbestrahlung kombiniert bis zu einer Gesamtdosis von 39 Gy/B-Linie.

Vor 1977 wurden anstelle der HDR-Kontaktbestrahlung analog intravaginale Radium-einlagen postoperativ bzw. intrauterine (Packmethode) bei der primären Strahlentherapie gegeben. Die Isodosenverläufe der LDR und HDR Technik zeigten weitreichende Überein-stimmungen. Wie aus der Tabelle I ersichtlich, konnten wir im Vergleich zur Radiumtherapie bei primärer Bestrahlung die 5-Jahresüberlebensrate um $9 \%$ von $66 \%$ auf $75 \%$. bei postoperati-ver Bestrahlung um 5\% von $83 \%$ auf $88 \%$ im Stadium I verbessern. Beim Stadium II gelang uns eine Anhebung der Heilungsrate bei primärer Strahlentherapie um 16\% von 53\% auf 69\% und bei einer auf Operation folgenden Nachbestrahlung um $12 \%$ von $61 \%$ auf $73 \%$. Bei fortgeschrittenen Karzinomen ist ein sicherer Vergleich der Werte nicht möglich. 


\section{Freie Vorträge}

40

Tabelle I. Korpuskarzinom: Vergleich der 5-Jahresüberlebensraten zwischen 643 mit konventioneller HDR-Radium-Technik von 1968 bis 1977 und 282 mit HDR-Afterloading-Technik von 1977 bis 1981 an der UFK Gießen behandelten Patienten

Schwere posttherapeutische Komplikationen in Form von Blasen-Scheiden- und Rek-tumScheiden-Fisteln mußten in $2 \%$ verzeichnet werden. Sie waren auf eine niedrige Fraktionierung bei hoher Einzeldosis in der ersten Zeit der HDR-Bestrahlung zurückzufüh-ren und konnten später nach Korrektur dieser Parameter bei der Primärtherapie gänzlich vermieden werden. Lokalrezidive traten in 3\%' nach der Bestrahlung auf. Auch hier konnte die Einführung niedriger Einzeldosen bei stärkerer Fraktionierung die Quote der Lokalrezidive deutlich vermindern. Nach unseren Erfahrungen kann man daher schließen, daß bei der Therapie des

Korpuskarzinoms

Die HDR-Aftrerloading-Technik im Vergleich zur konventionellen Radiumbe-handlung die Heilungsergebnisse verbessern kann und

Die Rate der Lokalrezidive und schweren Komplikationen durch mehr Fraktionen mit niedriger Einzeldosis verringert wird. 